\title{
REFERENCES
}

1. A. Bialynicki-Birula, On Galois theory of fields with operators, Amer. J. Math. 84 (1962), 89-109.

2. E. R. Kolchin, Galois theory of differential fields, Amer. J. Math. 75 (1953), 753-824.

3. - Algebraic matric groups and the Picard-Vessiot theory of homogeneous linear ordinary differential equations, Ann. of Math. (2) 49 (1948), 1-42.

University of California, Berkeley and

Institute of Mathematics, P.A.N., Poland

\section{ON A REALIZATION OF A COMPLEMENTED ALGEBRA}

\section{PARFENY P. SAWOROTNOW}

In this note we intend to show that each simple complemented algebra is isomorphic to an algebra described in the example below (as in [6] we use the term "simple" to mean "simple and semisimple"). This paper can be considered as a continuation of [5] and [6].

In the example below (and in the proof of the theorem after it) we use terms "summable" and "integrable" in the sense defined in Chapter III of [3].

ExAmple. Let $(S, \mu)$ be a measure space. Let $K(s)$ be a real-valued function defined on $S$ and having the following properties:

(i) $K(s)$ is finite almost everywhere,

(ii) there exists a positive number $a$ such that $a \leqq K(s)$ for each $s \in S$,

(iii) the restriction of $K(s)$ to any summable subset of $S$ is integrable (in particular $K(s)$ may be integrable).

Let $A$ be the set of all complex-valued members $x$ of $L^{2}(S \times S, \mu \times \mu)$ such that $\iint|x(t, s)|^{2} K(s) d t d s$ is finite. Then $A$ is a complemented algebra in the scalar product $(x, y)=\iint x(t, s) \bar{y}(t, s) K(s) d t d s$ and the multiplication $(x y)(t, s)=\int x(t, r) y(r, s) d r$ (we consider pointwise addition and pointwise multiplication with a scalar). If $K(s)$ is bounded above then $A$ is well complemented. Condition (ii) implies continuity of the multiplication (in both factors simultaneously); if $a=1$ then $\|x y\| \leqq\|x\|\|y\|$.

Presented to the Society, August 24, 1956, under the title Diagonalization of a complemented algebra; received by the editors July 15, 1963. 
TheOREM. Every simple complemented algebra $A$ is isomorphic to an algebra described in the example above. If $A$ is separable (if the underlying Hilbert space is separable) then the function $K(s)$ in the example above is integrable. If $A$ is well complemented, then the function $K(s)$ is bounded above.

Proof. We proved in [6, Theorem 3] that there exists a Hilbert space $H$ and a positive self-adjoint operator $\alpha$, having a bounded inverse $\beta$, such that the algebra $A$ is isomorphic to the algebra of operators $x$ of the Hilbert-Schmidt type, acting on $H$, such that $x \alpha$ is a Hilbert-Schmidt operator. The operator $\alpha$ is not bounded in general, but its domain is dense in $H$ ( $\alpha$ is bounded if and only if $A$ is well complemented). The operator $\beta\left(=\alpha^{-1}\right)$ is bounded, self-adjoint and positive (in the sense that $0<(\beta(h), h)$ for each $h \neq 0$ in $H$ ). Consider the algebra $B$ generated by the operators $\beta$ and the identity. By Theorems 61 and 64 of [4] there exists a family $\left\{M_{\gamma}, \mu_{\gamma}\right\}_{\gamma \in \Gamma}$ of measure spaces and a family $\left\{f_{\gamma}\right\}_{\gamma \in \Gamma}$ of bounded, real-valued, almost everywhere positive summable functions such that

$$
H=\sum_{\gamma \in \Gamma} L^{2}\left(M_{\gamma}, \mu_{\gamma}\right)
$$

and $(\beta(\phi))(m)=f_{\gamma}(m) \phi(m)$ for every $\phi \in L^{2}\left(M_{\gamma}, \mu_{\gamma}\right)$ (each $f_{\gamma}(m)$ is defined on $\left.M_{\gamma}\right)$. The summability of $f_{\gamma}$ follows from the fact that the measure on $M_{\gamma}$ is finite (a consequence of the fact that algebra $B$ has an identity) and that $f_{\gamma}(m) \leqq\|\beta\|$ for each $m \in M$ and each $\gamma \in \Gamma$.

Now let us consider the spaces $M$ as all different (and disjoint). It could be done, for example, by identifying each point $m$ in $M_{\gamma}$ with the ordered pair $(m, \gamma)$; however we do not want to complicate the notation. Let $S$ be the set-theoretic union of all members of the family $\left\{M_{\gamma}\right\}_{\gamma \in \Gamma}$ (formally $S=\left\{(m, \gamma) \mid \gamma \in \Gamma, m \in M_{\gamma}\right\}$ ); then each $M_{\gamma}$ is a subset of $S$.

We introduce an integral on $S$ as follows: A positive real-valued function $g$ defined on $S$ is summable if there exists a countable subset $\Gamma_{g}=\left\{\gamma_{1}, \gamma_{2}, \cdots, \gamma_{k}, \cdots\right\}$ of $\Gamma$ such that restriction of $g$ to $M_{\gamma}$ vanishes almost everywhere for $\gamma \notin \Gamma_{o}$ and such that $\sum_{k=1}^{\infty} \int g_{\gamma_{k}}(m) d m$ converges $\left(\int g_{\gamma}(m) d m\right.$ denotes the integral over $M_{\gamma}$ with respect to the measure $\left.\mu_{\gamma}\right)$. We define $\int g(m) d m=\sum_{k=1}^{\infty} \int g_{\gamma_{k}}(m) d m$. The realvalued function $g$ is summable if both $g^{+}$and $g^{-}$are summable; we define $\int g(m) d m=\int g^{+}(m) d m-\int g^{-}(m) d m$. We define summability of a complex-valued function in the usual fashion by considering its real and imaginary parts.

Then it is not difficult to show that $H=L^{2}(S)$. We define the function $\beta(m)$ on $S$ setting $\beta(m)=f_{\gamma}(m)$ if $m \in M_{\gamma}$. Then the restriction 
of $\beta$ to each summable subset $S$ is integrable. To see this, consider a summable set $T$. Then there exists a countable set $\Gamma_{0}=\left\{\gamma_{1}, \gamma_{2}, \cdots\right.$, $\left.\gamma_{n}, \cdots\right\}$ of $\Gamma$ such that $T \cap M_{\gamma}$ is a set of measure zero for each $\gamma \notin \Gamma_{0}$ and so that $\sum_{k=1}^{\infty} \mu_{\gamma_{k}}\left(T \cap M_{\gamma_{k}}\right)$ converges. If $\phi_{n}$ is the characteristic function of $\bigcup_{k=1}^{n}\left(T \cap M_{\gamma_{k}}\right)$ then $f_{n}(m)=\phi_{n}(m) \beta(m)$ is summable and $f_{n}(m)$ increases almost everywhere to the restriction of $\beta(m)$ to $T$. Also the restriction of $\alpha(m)=1 / \beta(m)$ and $K(m)=\alpha^{2}(m)$ to any summable subset of $S$ is integrable. Also $K(m) \geqq\|\beta\| \|^{-2}$ and $K(m)$ is finite almost everywhere (otherwise one could find $h \in H$ with $h \neq 0$ such that $\beta(h)=0$ ).

The rest of the theorem is easy to establish (note that $K(s)$ of the example corresponds to the function $\left.K(m)=\alpha^{2}(m)\right)$.

At the present time, the author would like to take the opportunity to make a correction in the announcement of his result, Diagonalization of a complemented algebra, Abstract 708t, Bull. Amer. Math. Soc. 62 (1956), p. 579. The term "measurable" was used in the sense of Loomis [3, p. 36]. Unfortunately, the author neglected to notice that this definition is valid only in the presence of an additional condition which does not have to be true in our case. The result stated in the abstract is still valid if we assume separability. In general the result should be stated in the way it was done in the theorem above.

In order to see that $K(s)$ does not have to be integrable consider $L^{2}(J)$ where $J$ is any noncountable set with discrete measure. Then any constant function defined on $J$ is not integrable. Take $K(s)$ to be identically equal to any positive number greater than 1 .

\section{REFERENCES}

1. W. Ambrose, Structure theorems for a special class of Banach algebras, Trans. Amer. Math. Soc. 57 (1945), 364-386.

2. P. R. Halmos, Measure theory, Van Nostrand, New York, 1950.

3. L. H. Loomis, An introduction to abstract harmonic analysis, Van Nostrand, New York, 1953.

4. G. W. Mackey, Commutative Banach algebra, Lecture Notes, Harvard Univ., 1952, Livraria Castelo, Rio de Janeiro, 1959.

5. P. P. Saworotnow, On a generalization of the notion of $H^{*}$-algebra, Proc. Amer. Math. Soc. 8 (1957), 49-55.

6. - On the imbedding of a right complemented algebra into Ambrose's $H^{*}$ algebra, Proc. Amer. Math. Soc. 8 (1957), 56-62.

The Catholic University of America 